\title{
Using ALFA Detectors for the LHC Beam Background Measurements*
}

\author{
Bartosz Dziedzic on behalf of the ATLAS Collaboration ${ }^{\dagger}$ \\ Institute of Nuclear Physics Polish Academy of Sciences \\ Radzikowskiego 152, 31-342 Kraków, Poland
}

\begin{abstract}
This paper presents background measurements performed during special runs with high $\beta^{*}$ optics at injection energy using ALFA detectors in the ATLAS experiment at the LHC. Such special runs require specific collimation scheme which reduces beam-induced background. Hence, complex preparation including simulations and tests had to be performed. This document discusses results of tests with different collimation schemes and background measurement during final data taking. In particular one of the runs was devoted to test a new type of collimators based on crystal channelling effect, that were used during physics data taking for the first time at the LHC.
\end{abstract}

Keywords: ATLAS experiment, ALFA detector, beam background, collimation, LHC

\section{INTRODUCTION}

Forward detectors at the LHC are designed to register protons scattered at very small angles. Such protons escape the acceptance of the main LHC detectors through the beam pipe and traverse the machine magnetic lattice accompanying the proton bunches. To measure precisely the scattering angles of such protons the detectors have to be placed in the immediate vicinity of the proton beam. Typically, the distance is of the order of a few millimetres from the beam centre. Therefore, such detectors are exposed to backgrounds that are in-time with the beam - coherent backgrounds called the beam halo. On the other hand, the forward detectors can be used as a great tool for measurements of such backgrounds.

The paper is organised as follows. The next section introduces the ALFA detectors at the ATLAS experiment. Section 3 describes the beam conditions during the test and physics runs. The proton beam collimation is discussed in Section 4. Section 5 gives the details of the performed test runs, while Section 6 describes the physics runs. The observations are discussed in Section 7 which is followed by the summary.

\section{ALFA DETECTORS AND THE TRIGGER SYSTEM}

ALFA (Absolute Luminosity For ATLAS) ${ }^{1}$ is a forward detector that is a subsystem of the ATLAS Experiment. ${ }^{2}$ This detector is designed to measure the absolute luminosity of the LHC, making use of the elastically scattered protons. It is an ensemble of eight detectors installed inside four Roman Pot stations placed symmetrically at a distance of about $240 \mathrm{~m}$ from the ATLAS interaction point (IP). The closer (or NEAR) stations are located about 237 meters away from the IP, while the further (FAR) ones are positioned 245 meters away from the IP. The Roman Pot technology allows insertion of the detector into the beam pipe and positioning it in the vicinity of the proton beam. The detectors move along the vertical direction (perpendicular to the LHC ring plane) so that there are a few milimeters between the detector and the beam centre. This distance is measured in units of the local beam width. Due to the operation very close to the proton beam, the Roman Pots are controlled only from CERN Control Center (CCC) and only in close co-operation of the LHC operators and Collimation Working Group (CWG).

Each Roman Pot station houses an upper and a lower detector. Each detector consists of two parts - a Main Detector (MD) and an Overlap Detector (OD). The MDs are used for tracking protons scattered at very small angles, while the ODs are used to determine the relative alignment between the upper and lower detectors. The detectors are shown in Fig. 1.

Presented at XLIV-th IEEE-SPIE Joint Symposium Wilga 2019 by B. Dziedzic: e-mail bartosz.dziedzic@ifj.edu.pl

Copyright 2019 CERN for the benefit of the ATLAS Collaboration. Reproduction of this article or parts of it is allowed as specified in the CC-BY-4.0 license 

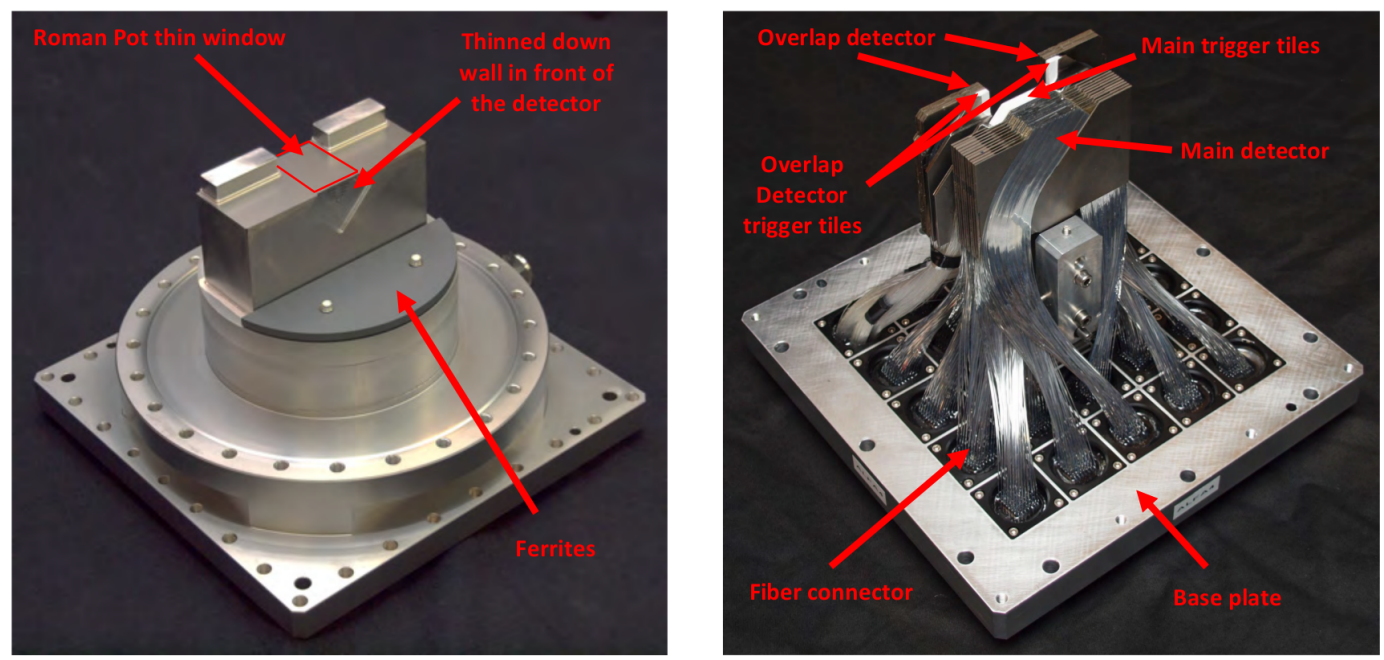

Figure 1. The Roman Pot (left picture) and ALFA detector including MD and OD (right picture). ${ }^{3}$

The Main Detector consists of ten double layers of scintillating fibres oriented alternatingly at the angle of $\pm 45^{\circ}$ with respect to the vertical direction. Each layer contains 64 squared fibres coated with a thin layer of aluminium. The size of a single fibre is $0.5 \times 0.5 \mathrm{~mm}^{2}$, and the resolution of the track position measurement is about $30 \mu \mathrm{m}$. The Overlap Detectors consist of two parts placed symmetrically on the MD sides. Each of them contains three layers of staggered scintillating fibres, 32 fibres each. The fibres are the same as those in MDs.

Behind each MD there are two $3 \mathrm{~mm}$ thick scintillating tiles used for triggering. Only a coincidence of both tiles generates the trigger signal. For the ODs, only single tiles are used for triggering. As each detector can send only a single trigger signal at the time, the prioritisation of triggers is applied - MD has higher priority than OD, thus if OD and MD generate triggers simultaneously, only MD trigger is sent to ATLAS Central Trigger Processor (CTP) over the $280 \mathrm{~m}$ long cable. ${ }^{4}$

The time of flight of a scattered proton is $800 \mathrm{~ns}$. The trigger signal propagation takes $1030 \mathrm{~ns}$ and its processing on the detector and the CTP side takes $423 \mathrm{~ns}$. This sums up to $2253 \mathrm{~ns}$ in total while the maximum ATLAS latency is $2175 \mathrm{~ns}^{3}{ }^{3}$ To solve this problem a new VME module was developed - ALFA_CTPIN. This module is responsible for fast decoding of the trigger signals and reduces the total time by $135 \mathrm{~ns}$ resulting in the total time of $2118 \mathrm{~ns}^{3}{ }^{3}$ ALFA_CTPIN required firmware upgrade and clock frequency increase of the digital front-end, thus latency was reduced by $12.5 \mathrm{~ns}$. It also procvided an opportunity to reduce LEMO cables' length saving another $21 \mathrm{~ns}$. $102.5 \mathrm{~ns}$ was saved directly on signal processing in ALFA_CTPIN module. The sources of the latency are presented in Table 1.

ALFA_CTPIN allows a measurement of the frequency of the produced trigger signals, providing information within $1.5 \mathrm{~s}$ long periods of time. Moreover, the rates are measured for each LHC bunch crossing separately. Therefore, it may be used as a basic tool for the machine background determination. ${ }^{5}$

\section{BEAM CONDITIONS FOR ALFA RUNS}

The data for both the test and physics runs were taken at the LHC proton beam injection energy of $450 \mathrm{GeV}$. The chosen optics used different betatron function ${ }^{\ddagger}$ values in $\mathrm{x}$ and $\mathrm{y}$ directions: $\beta_{x}^{*}=50 \mathrm{~m}, \beta_{y}^{*}=100 \mathrm{~m}$. During the test runs, both beams contained four colliding proton bunches. The physics runs used either six colliding isolated bunches or five colliding isolated bunches with a sixth non-colliding one. The non-colliding bunch was used to estimate the beam-related background (section 6). During data taking the ALFA detectors were positioned at the distance of $3 \sigma=4.5 \mathrm{~mm}$ from the beam center. In all runs, the beams collided head on.

\footnotetext{
${ }^{\ddagger}$ The betatron measures the distance after which the beam doubles its transverse dimensions. It is a function of the distance along the beam orbit.
} 
Table 1. Comparison of latency before and after ALFA_CTP installation

\begin{tabular}{|c|c|c|c|}
\hline \multicolumn{2}{|c|}{ Run2 before ALFA_CTPIN installation } & \multicolumn{2}{|c|}{ Run2 after ALFA_CTPIN installation } \\
\hline Source of latency & Latency $[\mathrm{ns}]$ & Source of latency & Latency $[\mathrm{ns}]$ \\
\hline Time of flight & 800 & Time of flight & 800 \\
\hline Scintillator & 3 & Scintillator & 3 \\
\hline Photomultiplier & 6 & Photomultiplier & 6 \\
\hline LEMO-cables (tunnel) & 25 & LEMO-cables (tunnel) & 10 \\
\hline MAROC2 (analog FE) & 13 & MAROC2 (analog FE) & 13 \\
\hline Digital Front End & 50 & Digital Front End & 37.5 \\
\hline Air-core-cable & 1030 & Air-core-cable & 1030 \\
\hline LEMO-cables (USA15) & 15 & LEMO-cables (USA15) & 10 \\
\hline MDOD separation & 60 & \multirow{4}{*}{ ALFA_CTPIN } & \multirow{4}{*}{37.5} \\
\hline NIM to LVDS & 5 & & \\
\hline CTPIN logic & 25 & & \\
\hline CTP PITbus upgrade & 50 & & \\
\hline CTP functionality & 125 & CTP functionality & 125 \\
\hline CTP OUT upgrade & 25 & CTP OUT upgrade & 25 \\
\hline New position of outer stations & 21 & New position of outer stations & 21 \\
\hline TOTAL & 2253 & TOTAL & 2118 \\
\hline
\end{tabular}

\section{BEAM COLLIMATION SYSTEM DESIGN}

The trajectories of the LHC beams are controlled by a large number of superconducting magnets. This set of magnets is called the cold aperture. In some sections, where high radiation levels are reached, the machine uses classical high-temperature magnets - the warm aperture regions. As there are always some regular and inevitable beam losses, it is very important to limit them to the warm aperture regions. Thus, a complex beam collimation system is essential. One should notice that the collimation system operation leads to the background reduction and optimisation which are of primary importance also for the forward detectors. The CWG in cooperation with experimental groups works on simulations of different collimations schemes according to the requests. It should be stressed that in some situations (as in the case of the ALFA high $\beta^{*}$ runs) the physics background optimisation is a very complicated process and it may take long time to achieve an acceptable background level. ${ }^{6}$

The LHC tunnel hosts more than 100 collimators. The majority of them is installed within two machine sections - IR3 and IR7. Those are devoted to the off-momentum particles and the betatron cleaning, respectively. Some additional collimators are installed within the experimental insertions, transfer lines, beam dump and around injectors. ${ }^{7}$ A schematic layout of the LHC collimation system is presented in the Fig. 2.

Collimators are organized in a multistage system. This means that all collimators are divided into groups:

- primary (TCP - Target Collimator, Primary),

- secondary (TCSG - Target Collimator, Secondary, Graphite),

- tertiary (TCTP - Target Collimator, Tertiary, Pick-up),

- absorbers (TCL - Target Collimator, Long Absorber, TCLA - Target Collimator, Long, Active Absorber).

Each of the above groups is designed to fulfill a different task. TCPs, TCSGs and TCLAs are installed in the cleaning insertion regions (IR3, IR7). Primary collimators are those closest to the beam and are cleaning the primary beam halo coming from regular beam losses. Primary collimators also cause the secondary beam halo and the hadron showers (see Fig. 3). The secondary collimators are devised to clean the secondary halo. They are closed to distances not smaller than those for the primary halo collimators. Naturally, the TCSGs scatter 


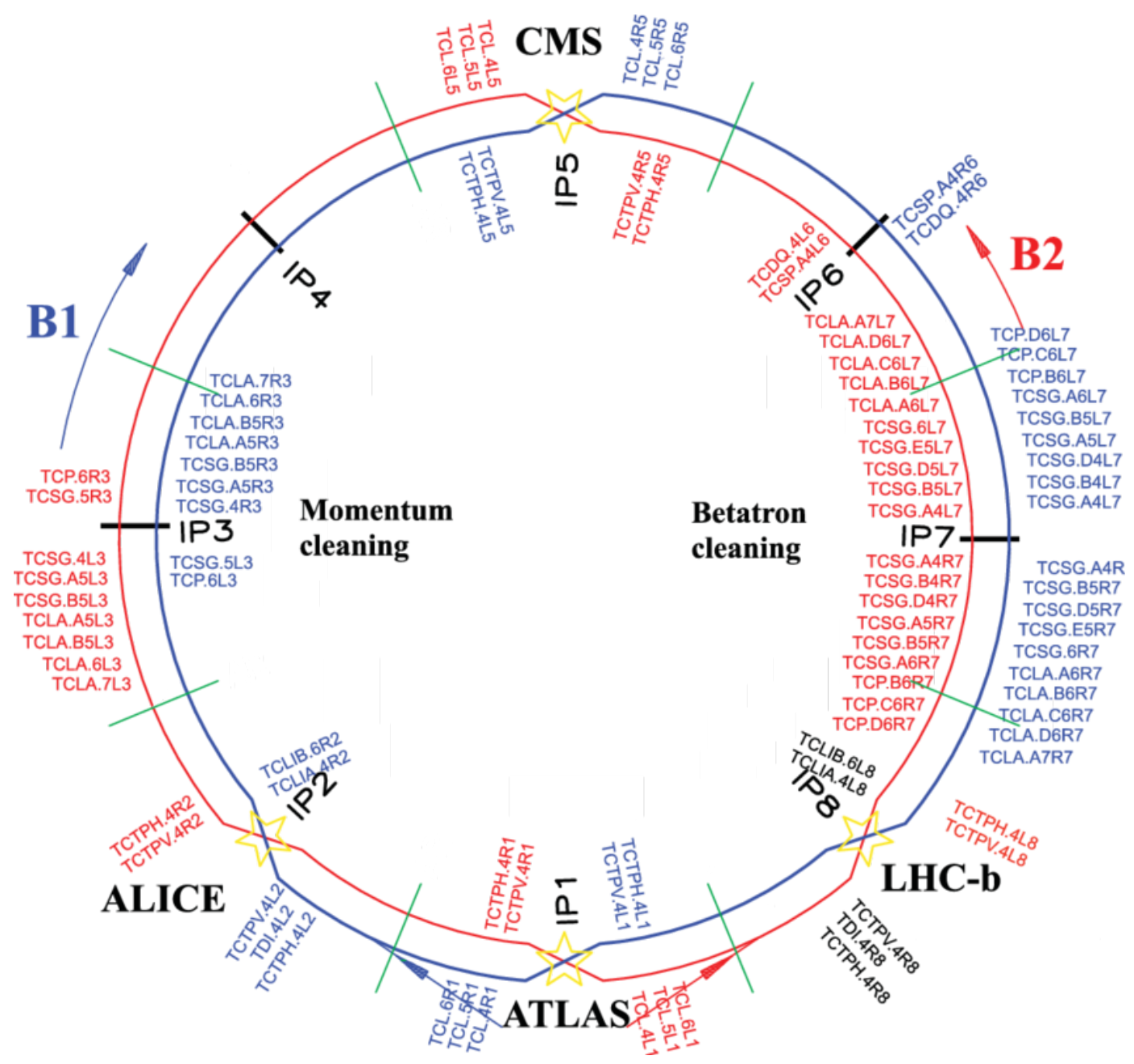

Figure 2. Layout of the collimation system. ${ }^{7}$

stray protons inducing thus the tertiary beam halo and hadronic showers which are caught by the absorbing collimators (TCLAs). The tertiary collimators are installed upstream of the experimental areas to protect their equipment and magnets. The physics debris absorbers (TCLs) are installed downstream of the experimental areas. ${ }^{7}$

\section{ALFA TEST RUN SETUP}

The aim of the test was to find the optimal collimation scheme allowing for efficient data taking, providing low beam-induced background level. During the test runs, the unprescaled rates of the following triggers were monitored on-line: triggers from each MD detector, an elastic trigger and a systematic trigger. The elastic trigger uses a simple logic to estimate the rate of elastic events (see Fig. 4). The systematic trigger delivers an estimation of the background level.

The first test run was performed on 8th of November 2017. The detectors were located $3 \sigma$ from the beam centre. Each of seven schemes started with a beam scraping lasting about 3-5 minutes. During beam scraping, the TCTPs were inserted into $3 \sigma$ position until the readings of Beam Loss Monitors (BLMs) stabilised. Meanwhile, the TCLAs and TCTPs were set in the positions according to prepared schemes. Then, the TCPs were retracted to the $5.5 \sigma$ position. For each test, data were collected over a period of 30 minutes. 




Figure 3. Multistage collimation scheme. ${ }^{7}$

After every scraping procedure, the MD trigger rate increased very quickly. It reached a level of $10 \mathrm{kHz}$ in about one minute. The elastic trigger rate was $50-200 \mathrm{~Hz}$, depending on the chosen configuration and the TCLAs and TCTPs settings, while the expected value from simulations was of order of $10 \mathrm{~Hz}$.

The second test run was performed two weeks later. Six collimator settings schemes were tested. Apart from a new collimation schemes including scraping up to $2.0 \sigma$ and the Roman Pot positions in $3.5 \sigma$, an increase of the $\mathrm{RF}$ voltage from 6.6 to $12 \mathrm{MV}$ and different tunes of the octupole magnets were tested. Only one of the tested schemes looked promising in the on-line monitoring. This scheme was repeated for the $3 \sigma$ Roman Pot positions. The MD trigger rate was lower than $500 \mathrm{~Hz}$ up to 15 minutes after the scraping while the elastic trigger rate was below $10 \mathrm{~Hz}$. Unfortunately, further off-line analysis showed that none of the schemes could be used for physics data taking because the background levels were still too high.

The third test run was performed in May 2018. Three schemes were devised. For that test the TCPs were in fixed positions of $3 \sigma$ and during the scraping the TCTPs and TCLAs were moved to the $2 \sigma$ positions. After the scraping the TCTPs and TCLAs were retracted to $2.5 \sigma$ positions. The RF voltage was increased from the nominal value of $6 \mathrm{MV}$ to $12 \mathrm{MV}$ to avoid debunching effects. The elastic trigger rate stayed below the expected level of $10 \mathrm{~Hz}$ for only a few seconds. Two minutes after the scraping, the elastic trigger rate exceeded few hundred Hz. The MD trigger rates exceeded $10 \mathrm{kHz}$ within less than three minutes. Further off-line analysis confirmed high level of the irreducible background proving that also these schemes were not proper for the physics data taking.

The fourth test was performed in October 2018. For that test all vertical collimators were inserted to positions 2.5-3.0 $\sigma$, while the RF voltage was reduced to $4 \mathrm{MV}$. The elastic trigger rate was changing between $10 \mathrm{~Hz}$ and $15 \mathrm{~Hz}$ for almost 40 minutes, while the MD trigger rate of each of the detectors did not exceed $1 \mathrm{kHz}$. Further off-line analysis demonstrated that this scheme can be used for the physics data taking.

\section{PHYSICS DATA TAKING SETUP}

To collect physics data, the fourth set of settings discussed above were used (i.e. multistage collimation). They are referred in the following as Setting 1. In addition, the so-called crystal channelling collimation tests were performed, and these settings are called Setting 2. Table 2 presents the collimators' positions during data taking with the collimation policy as described according to collimation Settings 1 and Setting 2.

LHC Fill 7284 was devoted to the multistage collimation scheme, and the procedure leading to the data taking was as follows. Once the beam was set by the LHC operators, the initial scraping was performed - the 
a)

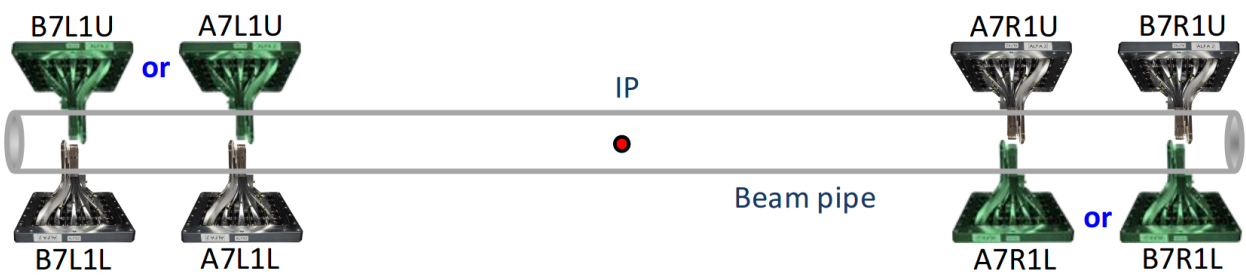

b)

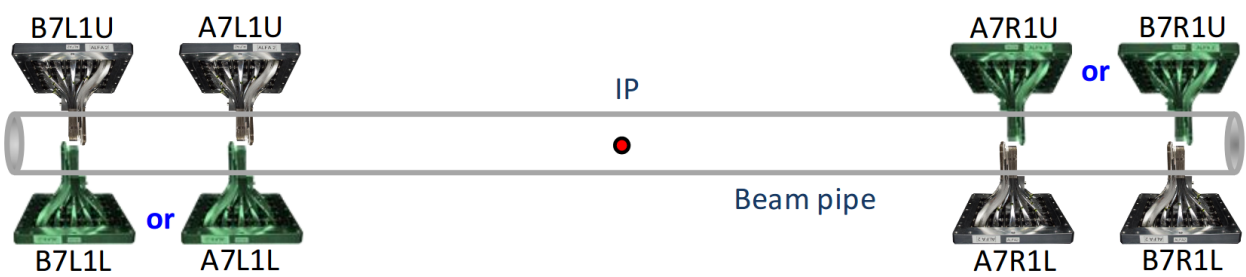

c)

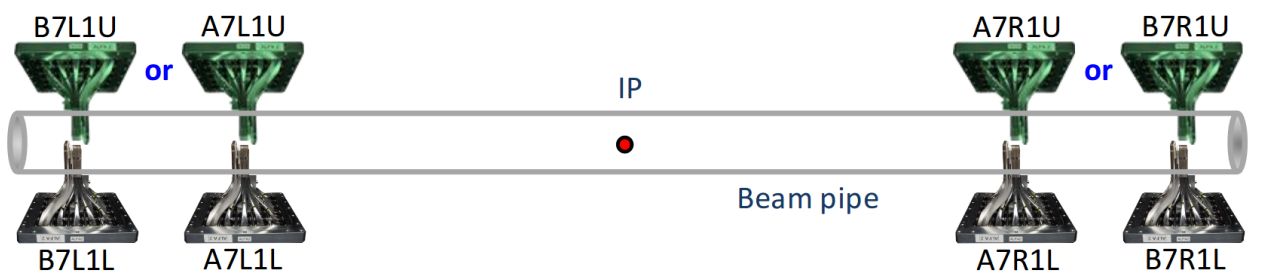

d)

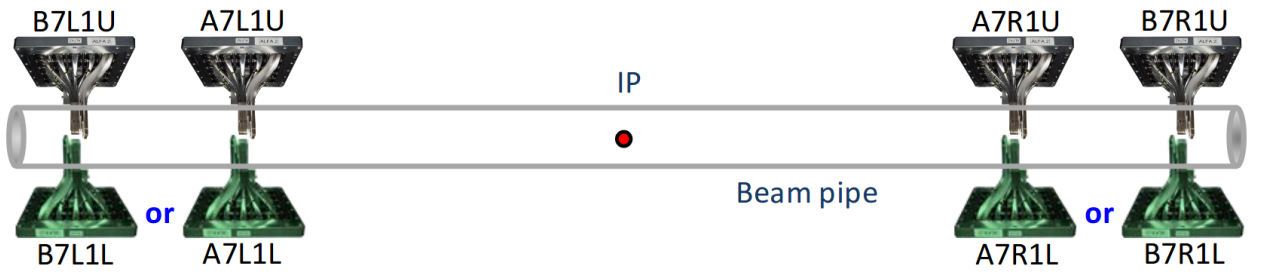

Figure 4. Elastic trigger logic and the systematic trigger logic. ${ }^{8}$ Logic a) and b) correspond to elastic trigger patterns, while c) and d) show the systematic trigger patterns.

TCLA.A6 and TCP.C6 were moved to $2.0 \sigma$ and $3.0 \sigma$ positions, respectively, and then the positions of the other collimators were set according to the values listed in Table 2. When the BLM rates stabilised, the ALFA Roman Pots were moved into their operation positions, i.e. 3.0 $\sigma$. Then the TCLA.A6 and TCP.C6 collimators were retracted to the data taking position $-4.0 \sigma$ and $2.5 \sigma$ respectively (Table 2 ). During the data taking the elastic and systematic trigger rates were monitored. The systematic trigger rate rose slowly, and when it reached the elastic one, approximately every hour, beam re-scraping was required. When the elastic rate after the re-scraping fell below $10 \mathrm{~Hz}$, the beam was dumped and refill of the LHC was requested. The data-taking run took about four hours.

LHC Fill 7289 was devoted to the crystal collimation scheme. The initial scraping procedure was similar to that mentioned above. After initial scraping, the TCLA.A6 collimators were retracted from $2.5 \sigma$ to $2.7 \sigma$ and the crystal collimators were inserted to the $2.5 \sigma$ position. After all the parameters stabilised the Roman Pots were inserted to the $3.0 \sigma$ positions. Positions of the collimators for data taking with the use of crystal collimators are presented in Table 2. The systematic trigger rate increased much more slowly than in the multistage collimation scheme case and the data taking lasted for over three hours without a re-scraping request. 
Table 2. Collimators positions in $\sigma$ for runs with multistage collimation scheme (Setting 1) and for run with crystal collimation scheme (Setting 2).

\begin{tabular}{|l|c|c|c|c|}
\hline Collimator & Plane & IR & Setting 1 & Setting 2 \\
\hline TCLA.A6 & $\mathrm{V}$ & 7 & 2.5 & 2.7 \\
TCLA.A5 & $\mathrm{V}$ & 3 & 2.7 & 2.7 \\
TCTPV.4L2 & $\mathrm{V}$ & 2 & 2.7 & 2.7 \\
TCTPV.4R8 & $\mathrm{V}$ & 8 & 2.7 & 2.7 \\
TCTPV.4L2 & $\mathrm{V}$ & 1 & 2.7 & 2.7 \\
TCTPV.4R8 & $\mathrm{V}$ & 5 & 2.7 & 2.7 \\
TCLA.C6 & $\mathrm{V}$ & 7 & 2.7 & 2.7 \\
TCP.D & $\mathrm{V}$ & 7 & 3.0 & 3.0 \\
\hline TCP.6 & $\mathrm{H}$ & 3 & 5.3 & 5.3 \\
All TCSGs & $\mathrm{H}$ & 3 & 6.3 & 6.3 \\
All TCSGs & $\mathrm{H}$ & 3 & 9.0 & 9.0 \\
TCP.C6 & $\mathrm{H}$ & 7 & 4.0 & 4.0 \\
TCSG.B4 & $\mathrm{H}$ & 7 & 5.0 & 5.0 \\
TCSG.6 & $\mathrm{H}$ & 7 & 5.0 & 5.0 \\
TCLA.B6 & $\mathrm{H}$ & 7 & 8.0 & 8.0 \\
TCLA.A7 & $\mathrm{H}$ & 7 & 8.0 & 8.0 \\
\hline Crystal & $\mathrm{V}$ & 7 & $\mathrm{n} / \mathrm{a}$ & 2.5 \\
\hline Roman Pots & $\mathrm{V}$ & $1 / 5$ & 3.0 & 3.0 \\
\hline
\end{tabular}

\section{BACKGROUND MEASUREMENT}

During physics data taking with the multistage collimation, each beam contained an additional non-colliding isolated bunch to measure the beam-induced background. This approach was repeated for the run with the crystal collimation scheme.

During the run with the multistage collimation scheme, the rates seen by the detectors on one side of the IP were comparable. However, the ratio of the readings on the left and right sides of the IP was nearly constant and close to 2. The interpretation was that beam2 (Fig. 2) gave twice as much background as beam1. The beam-induced background was growing constantly over time. The off-line analysis showed the irreducible elastic background contribution of about $0.5 \%$.

During the run with crystal collimation scheme, the ratio of the readings on the left and right sides of the IP was measured as well. Its value was close to the inverse of the one observed for the multistage collimation scheme. Moreover, the differences in the MD trigger rates between the detectors on the right side was much higher than that for ones on the left side, or for the multistage collimation. The rates from each of the Main Detectors on the left side of the Interaction Point were almost the same.

It is interesting to point out that for these runs, contrary to those with the multistage collimation scheme, the difference between the beams was observed not only in the trigger rates, but also in the spatial distributions of the measured tracks. The off-line analysis showed that the irreducible background for crystal is about $1.5 \%$, 3 times higher than for the multistage collimation.

\section{SUMMARY}

After almost one year of preparation, the ATLAS-ALFA detector took data at the LHC injection energy with high $\beta^{*}$ optics. For runs where multistage collimation was used, the background growth over time forced re-scraping roughly every hour. Beam-related background patterns look similar on each side of the IP. 
During the special runs the crystal collimators were tested. The growth of the trigger rates during those runs was much slower, thus re-scraping was not required for over three hours of data taking. There was a strong asymmetry in the beam-related background pattern, that requires further investigation.

Off-line analysis showed the irreducible elastic background was about $0.5 \%$ and $1.5 \%$ for multistage and crystal collimation respectively.

The usefulness of the crystal collimation scheme during high $\beta^{*}$ runs requires further off-line analysis. Conclusions from the discussed measurements will be used for preparation of the high $\beta^{*}$ runs expected in the future.

\section{REFERENCES}

[1] The ALFA Collaboration, "The ALFA roman pot detectors of ATLAS," Journal of Instrumentation 11(11), P11013 (2016).

[2] The ATLAS Collaboration, "The ATLAS experiment at the CERN large hadron collider," Journal of Instrumentation 3(08), S08003 (2008).

[3] Jakobsen, S., "ALFA latency for Run1 and expectations for Run2," Tech. Rep. ATL-COM-LUM-2013-018, CERN, Geneva (Jul 2013).

[4] Korcyl, K., "ALFA detector: timing and trigger," Proc. SPIE 89032-K1 (2013).

[5] Iwanski, W., Jakobsen, S., Korcyl, K., and Oechsle, J., "Functionality and performance of the ALFA_CTPIN module," PoS TWEPP-17, 142 (2017).

[6] Garcia Morales, H., Bruce, R., Burkhardt, H., Deile, M., Jakobsen, S., Mereghetti, A., and Redaelli, S., "Special Collimation System Configuration for the LHC High-Beta Runs," MOPML012 (2018).

[7] Redaelli, S., "Beam cleaning and collimation systems," CERN Yellow Report CERN-2016-002, pp.403-437 (2016).

[8] Jakobsen, S., "Commissioning of the Absolute Luminosity For ATLAS detector at the LHC," (2013). CERNTHESIS-2013-230. 